\title{
Oral symptoms significantly higher among long-term khat (Catha edulis) users in Ethiopia
}

\author{
Ayalew Astatkie ${ }^{1}$, Meaza Demissie ${ }^{2}$, Yemane Berhane ${ }^{2}$, Alemayehu Worku²,3 \\ ${ }^{1}$ School of Public and Environmental Health, College of Medicine and Health Sciences, Hawassa University, Hawassa; ${ }^{2}$ Addis Continental \\ Institute of Public Health, Addis Ababa; ${ }^{3}$ School of Public Health, College of Health Sciences, Addis Ababa University, Addis Ababa, Ethiopia
}

OBJECTIVES: Associations between khat (Catha edulis) chewing and different adverse oral-dental health conditions have been reported, yet evidence is still lacking. This study was designed to investigate the association between long-term regular khat chewing and self-reported oral symptoms.

METHODS: A cross-sectional study was conducted on a sample of 1,255 university students in southern Ethiopia. Data on khat chewing status, a range of oral symptoms and other pertinent variables were collected using self-administered questionnaires. The association between long-term regular khat chewing and oral symptom count was investigated using negative binomial regression.

RESULTS: The mean oral symptom count among long-term regular khat chewers was 1.75 (standard deviation [SD], 2.18; standard error [SE], 0.31), whereas that among those who were not long-term regular khat chewers was 1.18 (SD, 1.68; SE, 0.10). After adjustment for other variables, long-term regular khat chewers had approximately $50 \%$ more oral symptoms than those who were not long-term chewers did (adjusted count ratio, $1.53 ; 95 \%$ confidence interval, 1.12 to 2.10 ).

CONCLUSIONS: Long-term khat chewing negatively affects the oral health of young university students.

KEY WORDS: Khat, Catha edulis, Oral health, University, Students

\section{INTRODUCTION}

Studies ranging from case-reports to controlled experiments investigating the association between khat (Catha edulis) chewing and oral and dental health have been performed. Periodontal pocketing [1,2], gum bleeding [1-3], and gingival recession [1-5] have been shown to occur at a higher rate among khat chewers compared to those who do not chew. A dose-response relationship between khat chewing and the aforementioned adverse outcomes has also been reported [1]. In addition, khat

Correspondence: Ayalew Astatkie

School of Public and Environmental Health, College of Medicine and Health Sciences, Hawassa University, P.O. Box 1643, Hawassa, Ethiopia

Tel: +251-911739694, Fax: +251-462208755, E-mail: ayalewastatkie@gmail.com

Received: Jan 24, 2015, Accepted: Feb 19, 2015, Published: Feb 19, 2015

This article is available from: http://e-epih.org/

(C) 2015, Korean Society of Epidemiology

(C) This is an open-access article distributed under the terms of the Creative Commons Attribution License (http://creativecommons.org/licenses/by/3.0/), which permits unrestricted use, distribution, and reproduction in any medium, provided the original work is properly cited. chewers were found to have a higher odds of clinical attachment loss than non-chewers were [6]. Moreover, numerous other consequences associated with the khat chewing habit have been reported such as oral mucosa white lesions [7,8] (a doseresponse relationship [7]), plasma cell gingivitis [9], oral mucosal pigmentation [4], gingival pigmentation [10], oral mucosa ulceration [3], tooth staining [4], tooth loss [11], stomatitis [12], and temporo-mandibular joint disorder [13]. Caries, as measured by the decayed, missing and filled teeth index, have also been shown to be more likely among khat chewers compared to non-chewers [14]. Poor oral hygiene [3] and oral dryness [4] have been documented as occurring more among khat chewers, and a possible link between khat chewing and oral cancer [15] has also been suggested .

However, contrary to the findings above, several studies have documented a better periodontal health status among khat chewers relative to non-chewers $[16,17]$ or in the chewing side of the mouth compared to the non-chewing side [4]. In addition, absence of a significant difference between khat chewers and nonchewers has been demonstrated in terms of attachment loss [17], 
periodontal status as measured by community periodontal index [6], gingival inflammation [4], and oral leukoplakia [18].

Clearly, to date, the accumulated evidence is scanty, and the findings are contradictory. Besides, most previous studies have investigated the association between khat chewing and the occurrence of oral diseases, which were assessed objectively. However, there are oro-dental conditions that affect wellbeing and oral health care seeking behaviours, but which cannot be captured by traditional objective assessments (e.g., discomfort, pain, xerostomia, changes in the ability to taste, etc.) [19]. Such conditions can only be assessed subjectively through self-report. Hence, in the present study, the association between khat chewing and oral symptoms was investigated as an input to the ongoing scientific discourse on the association between khat chewing and oral health.

\section{MATERIALS AND METHODS}

\section{Study design and subjects}

This cross-sectional study was conducted among undergraduate students of Hawassa University in southern Ethiopia. At the time of data collection, the university had over 17,000 regular undergraduate students. More description of the university and the study population is available elsewhere [20].

Because we also had a plan to determine khat chewing practice and its associated factors among the student population, we restricted the sample population to only students who were in their second year of university and above. Those in their first year were not included because they were considered to be in a buffer period transitioning from being a high school student to a university student [20].

\section{Sample size and sampling}

Initially, the statistical method proposed to analyse the data was a Poisson regression; hence, the sample size required for a Poisson regression analysis was estimated using the software G*Power version 3.1 (Franz Faul, Universität Kiel, Kiel, Germany) [21]. However, Poisson regression was not performed, and the data were analysed using a negative binomial regression. The following assumptions were used in this calculation: a to-be-detected effect size (exp $\left.\left[\beta_{1}\right]\right)$ of 1.5 , alpha-value of 0.05 (two-tailed), power of $80 \%$, disease rate in non-chewers (base rate $\exp \left[\beta_{0}\right]$ ) of one (based on our best guess since there was no prior information available), fixed mean exposure time of one month and variance accounted for by other predictors $\left(\mathrm{R}^{2}\right.$ other $\mathrm{X}$ ) of 0.5 . Since there was no prior information on the variance, this value was used instead of the default zero to maximize the sample size. In addition, a binomial distribution for the exposure variable, long-term regular khat chewing (i.e., $\mathrm{X}$ distribution = binomial), was used. The parameter of the exposure variable (X-param $\pi$ ) was set at $28 \%$ based on the current prevalence of khat chewing among students of Axum University, Ethiopia [22]. This percentage was used as a proxy for the prevalence of long-term regular khat use since it has not been previously estimated. With these assumptions, the required sample size was found to be 377 . Considering a design effect of 1.5 and a non-response rate of $5 \%$, the final estimate was 595. As this study was part of a larger study designed to address other objectives that require a sample size as large as 1,290 , the sample size requirement to address our objective for this paper was fulfilled.

The sampling was accomplished using a two-stage stratified cluster sampling design. All undergraduate study programmes within the university constituted the primary sampling units. From each college/institute, two primary sampling units were selected using simple random sampling. Within each primary sampling unit, sections/classes formed the secondary sampling units. Then, the secondary sampling units were stratified by university year of study (second year, third year, fourth year and above). From each year of study, one secondary sampling unit was selected using simple random sampling. All of the students in the selected secondary sampling unit were included in the study since students within a university are assumed to be relatively homogenous.

\section{Data collection instruments and procedures}

The data collection was accomplished using a pre-tested selfadministered questionnaire. The questionnaire contained items to assess khat chewing behaviour and other relevant variables. It also contained nineteen items about oral symptoms such as toothache, sores/irritations in the mouth, gingival bleeding, oral malodour, dry mouth, teeth sensitive to heat/cold, and temporo-mandibular joint pain. These items were adapted from the study of Locker and Miller [23]. Such subjective oral health status assessment techniques have been used in different populations worldwide and have been found useful for epidemiological studies on the oral-health status of populations where clinical assessments of oral health are costly $[23,24]$.

Data were collected in a class after the necessary information on privacy and confidentiality and a comprehensive instruction on how to complete the questionnaire were provided to the study participants. While an Amharic-version of the questionnaire was mainly used for the data collection, the English version of the questionnaire was made available for those who reported difficulty understanding Amharic. All data collection sessions were facilitated by facilitators who were not part of the respective campus community. In addition, no one outside of the study was allowed to enter the classrooms at the time of data collection to ensure maximum privacy. 


\section{Variables}

The dependent variable was oral symptom count. Respondents were asked a series of nineteen questions on whether they had specific oral symptoms within the four weeks preceding the study. Then, a new variable was computed for the number of symptoms each respondent reported, and this new variable was used for subsequent analyses in this study as a count variable.

The major explanatory variable was long-term regular khat chewing defined as khat chewing for at least three years with a frequency of at least once a week. Regular chewing was categorized as yes or no. Other independent variables were also considered mainly to control for their potential confounding effects on the association between khat chewing and oral symptom count. These variables included cigarette smoking in the past 12 months (yes/no), usually using sugar in hot drinks (yes/no), frequency of soft drink consumption (less than once a week or never vs. one or more times per week), self-reported dental fluorosis (yes/no), tooth brushing (using a brush and tooth paste) frequency (not at all, less than once a day, one or more times per day), alcohol consumption in the past 12 months (yes/no), amount of monthly pocket money (in quintiles) and sex (female vs. male). Monthly pocket money was used as a proxy indicator of socioeconomic position.

\section{Statistical analysis}

To facilitate detection of data-entry errors, all data were entered twice using the software EPI Info version 3.5 (Centers for Disease Control and Prevention, Atlanta, GA, USA). Data entry errors were corrected by comparing the two independently entered datasets. Further data analyses were accomplished using Stata version 12 (StataCorp., College Station, TX, USA).

As is commonly encountered in self-administered questionnaires, we had some variables with item-missing data. Multiple imputation using chained equations [25] was performed in Stata to replace missing items and to retain as many of the respondents as possible in the analysis. Twenty imputations $(M=20)$ were created for each missing item using a burn-in period of 10 after checking the convergence of the imputation models using trace plots, as recommended in the Stata manual. Augmented regression was used to handle cases of perfect prediction when using categorical predictors [26]. Most of the important variables with item-missing data were successfully imputed. However, because the imputation models failed to converge, some variables were not imputed. Sensitivity analyses were employed by comparing the descriptive summaries of the imputed variables before and after imputation, and the analyses showed comparable results confirming the absence of any aberrations during the imputation [26].

The analysis was performed considering the complex sam- pling design. Hence, the stratification and clustering both in the first and second stages of sampling were taken into account. In addition, an analysis weight was employed that accounted for the unequal probability of selection in the first and second stage sampling units as well as a post-stratification adjustment factor based on the sex of the respondents [27].

To determine the association between long-term regular khat chewing and oral symptom count, a negative binomial regression was used instead of a Poisson regression since the assumption was not fulfilled due to the overdispersion of the data [28]. Univariable negative binomial regression using the major explanatory variable and each of the possible confounders was undertaken as the initial step. Then, variables with p-values of less than or equal to 0.25 in the univariable regression and those deemed important based on the literature were selected for inclusion into the initial multivariable model [27]. Serial refining and refitting of the initial multivariable model was also carried out. Variables with insignificant coefficients whose removal did not significantly affect the model as a whole and individual variables remaining in the model were deleted. Any improvement in the model was checked by including relevant interactions and higher-order terms one at a time, but none was found significant. Thus, the final model was a main-effects model containing the major explanatory variable (long-term regular khat chewing) and four other covariates (cigarette smoking, self-reported dental fluorosis, frequency of soft drink consumption and pocket money). After running the final model, the average adjusted predictions of oral symptom count by khat chewing status were estimated using Stata's user written command "mimrgns" [29]. The reproducibility of the results, which were based on multiply-imputed data, was assessed using Monte Carlo error estimates as per White et al.'s [25] guidelines. The Monte Carlo error estimates suggested that the results are quite reproducible. In other words, our results would be stable "across repeated uses of the same imputation procedure" [26].

\section{Ethics statement}

This study was approved by the institutional review board (IRB) of the College of Medicine and Health Sciences of Hawassa University, Hawassa, Ethiopia (IRB No. IRB/165/05). The respondents did not provide any personal identifiers on the questionnaires to ensure the anonymity of their responses. Respondents took part in the study voluntarily after a verbal informed consent that was endorsed by the aforementioned IRB.

\section{RESULTS}

Of the 1,290 proposed study participants, 1,255 completed a questionnaire resulting in a nonresponse rate of $2.7 \%$. Three 
respondents with missing information on the survey design variables were excluded by a case-wise deletion. Hence, the results in this article are based on 1,252 respondents with complete information (after imputation) for all variables used in this study.

Table 1. Oral symptoms according to khat chewing status among university students at Hawassa University, southern Ethiopia, June 2014

\begin{tabular}{lrr}
\hline & \multicolumn{2}{c}{$\begin{array}{c}\text { Long-term regular } \\
\text { khat chewing }\end{array}$} \\
\cline { 2 - 3 } Oral symptom & Yes & No \\
\hline Tooth ache & 16.6 & 11.6 \\
Pain in the jaw joint while chewing & 16.8 & 7.2 \\
Dryness of the mouth & 14.4 & 5.3 \\
Mouth ulcers & 13.6 & 4.5 \\
Burning sensation in the tongue or other parts of & 13.0 & 5.1 \\
$\quad$ the mouth & & \\
Pain in the jaw joint while opening the mouth wide & 12.8 & 4.9 \\
Bad breath & 10.3 & 11.1 \\
Unpleasant taste & 9.5 & 11.0 \\
Pain in the teeth with hot/cold foods/fluids & 9.4 & 9.3 \\
Bleeding gums & 9.2 & 14.0 \\
Shooting pain in the face or cheeks & 8.0 & 5.6 \\
Difficulty opening the mouth wide & 7.5 & 2.5 \\
Cold sores & 6.9 & 3.5 \\
Pain in the face in front of the ear & 6.5 & 5.0 \\
Sore gums & 5.2 & 5.6 \\
Clicking/grating noise in the jaw joint & 5.1 & 2.1 \\
Pain in the teeth with sweet foods & 3.8 & 5.7 \\
Changes in the ability to taste & 3.7 & 4.5 \\
Pain or discomfort from dentures & 1.0 & 1.0 \\
\hline
\end{tabular}

Values are presented as \%.
Of all the study participants, 7.6\% (95\% confidence interval $[\mathrm{Cl}, 3.7$ to $11.4 \%$ ) were long-term regular khat chewers. There was no significant difference in terms of mean age between those who were long-term regular khat chewers (mean, 22.3 years; standard deviation [SD], 1.48 years) and those who were not long-term regular khat chewers (mean, 21.4 years; SD, 1.54 years). However, more males $(9.9 \%)$ were long-term regular khat chewers than females were $(1.0 \%)$.

The oral symptoms reported more frequently by the longterm regular khat chewers included pain in the jaw joint while chewing $(16.8 \%)$, tooth ache $(16.6 \%)$, dryness of the mouth (14.4\%), mouth ulcers (13.6\%), and a burning sensation in the tongue or other parts of the mouth (13.0\%). Details of the oral symptoms by khat chewing status are shown in Table 1 .

The mean oral symptom count in the previous four weeks among the long-term regular khat chewers was 1.75 (SD, 2.18; standard error [SE], 0.31), whereas the mean among those who were not long-term regular khat chewers was 1.18 (SD, 1.68; $\mathrm{SE}, 0.10$ ). After adjustment for other covariates, long-term regular khat chewers had approximately $50 \%$ more oral symptoms than those who were not long-term regular khat chewers (adjusted count ratio, 1.53; 95\% CI: 1.12 to 2.10) (Table 2).

The average adjusted prediction of oral symptom count among the long-term regular khat chewers was $1.80(\mathrm{SE}=0.24)$. However, for those who were not long-term regular khat chewers, the average adjusted prediction was $1.17(\mathrm{SE}=0.09)$. Thus, if all study participants were treated as long-term regular khat chewers, the mean symptom count would be 1.80 , and if all were treated as not being long-term regular khat chewers, the mean symptom count would be 1.17 .

Table 2. Association between long-term regular khat chewing and oral symptom count among university students after adjustment for covariates, Hawassa University, southern Ethiopia, June 2014

\begin{tabular}{lcr}
\hline Variable $^{1}$ & Crude count ratio $(95 \% \mathrm{Cl})$ & Adjusted count ratio $^{2}\left(95 \% \mathrm{Cl}^{\prime}\right)$ \\
\hline Long term regular khat chewing $^{\text {No }}$ & 1.00 & 1.00 \\
Yes & $1.48(0.92,2.40)$ & $1.53(1.12,2.10)$ \\
Cigarette smoking in the past year & 1.00 & 1.00 \\
No & $1.28(0.76,2.15)$ & $1.21(0.76,1.94)$ \\
Yes & 1.00 & 1.00 \\
Self-reported dental fluorosis & $1.39(1.05,1.83)$ & $1.31(0.99,1.74)$ \\
No & 1.00 & 1.00 \\
Yes & $0.75(0.61,0.93)$ & $0.80(0.60,1.06)$ \\
Frequency of soft drink consumption & $0.91(0.83,0.99)$ & $0.91(0.82,1.00)$ \\
Less than once a week or never & & \\
One or more times per week & & \\
Monthly pocket money & &
\end{tabular}

$\mathrm{Cl}$, confidence interval.

${ }^{1}$ The initial multivariable model also included using sugar in hot drinks, tooth brushing, alcohol consumption and the sex of the respondent as covariates. However, they were subsequently deleted from the model because their coefficients were not significant and their removal did not significantly affect the model as a whole or the individual variables remaining in the model.

${ }^{2}$ Adjusted Wald test for all parameters: $F(5,3.6)=9.20, p=0.0332$.

${ }^{3}$ Entered as a continuous variable. 


\section{DISCUSSION}

In this study, the association between the number of self-reported oral symptoms and khat chewing in a population of university adolescents and young adults was investigated. Our data strongly suggest that chewing khat regularly for a long period is associated with higher self-reported oral symptoms. The average adjusted prediction of these symptoms is also clearly higher among those who were engaged in chewing khat regularly than those who were not.

The findings of the present study support a previous finding from Yemen among relatively older dental clinic patients amongst whom the khat chewers were found to have more oral complaints than the non-chewers were [3]. As mentioned above, khat chewing has been suggested to be associated with several oral morbidities. Different mechanisms by which khat chewing can bring about different adverse oral health outcomes have been suggested in the literature. First, the continuous mechanical friction and irritation on the cheeks, gums and other oral tissues may cause trauma to the oral structures and lead to different pathological changes in the oral cavity $[1,2,6,8]$. Al-Sharabi et al. [6] have pointed out that vertical impaction of khat fibres into the periodontium might lead to later periodontal abnormalities such as gum recession and periodontal pocketing. However, the possibility of check biting while chewing khat has not been mentioned elsewhere in the literature. Especially since the buccal mucosa becomes hypertrophic due to the chronic irritation [30] from khat chewing, the incidence of cheek biting is expected to be more likely in these individuals, causing considerable trauma to the buccal tissue. Cobián et al. [13] have documented a high prevalence of radiographic findings of condylar flattening and temporo-mandibular joint narrowing with associated joint pain and difficulty of mouth opening among khat chewers, which seem to be due to the mechanical effect of chronic khat chewing on the temporo-mandibular joint.

A second mechanism is thought to act through the toxic and irritating effects of the chemicals, especially the tannins, in khat $[8,31]$. Halbach [32] has noted that the frequent encounter of stomatitis among khat chewers could be attributed to the astringent tannins. Histopathological studies of the effects of khat on human oral mucosa under in vitro [31] and in vivo [33] conditions have also shown abnormal changes that could be attributed to the effects of the chemicals in khat as well as the mechanical effects. A third mechanism by which khat could adversely affect oral health is through the toxic effects of pesticides applied to the khat plant in the process of production. A previous study has shown that people chewing khat grown with pesticides have more chronic health complaints than do those chewing khat grown without pesticides [34]. As pesticides may permeate the khat tissues, washing the leaves before chewing might not remove these chemicals, and hence continue to cause an insult to oral health (and general health) [33,34]. A fourth and indirect mechanism might be the use of soft drinks and sugar crystals to counteract the bitter taste of khat. As these substances are cariogenic, they increase the likelihood of developing dental caries [14].

Hence, the occurrence of more oral complaints among regular khat chewers in the current study might be due to a combination of the different mechanisms through which khat chewing is expected to act to cause adverse oral health effects. However, the younger age profile of the study participants is worth noting. Among these khat chewers, the cumulative exposure to the khat chewing habit is expected to be much less than that of older adults and elderly people with the habit. Furthermore, advanced periodontal diseases are likely to be minimal in a younger population [35].

In addition, only self-reported information about oral symptoms was obtained in the present study. Some indicators of oral health disorders are detectable only through clinical oral examination such as gingival recession, bleeding on probing, mucosal white changes and others, which were not assessed here. Thus, if both the self-reported symptoms and the clinical signs were combined to form a sign/symptom count, the mean count in each khat chewing status group and the difference of the counts between the two groups could be larger.

Nevertheless, the fact that more oral symptoms were identified among the long-term khat chewers in the current study might indicate that more oral health care is needed among these students. However, oral diseases are among the most expensive conditions to treat worldwide [36]. In developing countries, the cost is unaffordable [36]. Given that Ethiopia has one dentist per one million people [37], oral health service would be not only unaffordable but inaccessible. Therefore, avoiding or reducing the exposure to the risk factors seem the more plausible option.

In conclusion, long-term regular khat chewing was found to increase the occurrence of oral symptoms among university students. Health professionals and other concerned bodies should consider khat chewing as a possible threat to oral health and take proper actions. Moreover, university students should be informed of the possible adverse oral health effects of khat chewing.

\section{ACKNOWLEDGEMENTS}

We acknowledge the kind help by our colleagues at the School of Public and Environmental Health of Hawassa University as well as the heads of departments/schools, the teachers and the Student Council members in the different campuses of Hawassa University. We remain indebted to the study participants who 
wilfully took part in the study.

Financial and material assistance for this study was obtained from Hawassa University and Addis Continental Institute of Public Health.

\section{CONFLICT OF INTEREST}

The authors have no conflicts of interest to declare for this study.

\section{REFERENCES}

1. Ali AA. Qat habit in Yemen society: a causative factor for oral periodontal diseases. Int J Environ Res Public Health 2007;4:243-247.

2. Astatkie A, Demissie M, Berhane Y. The association of khat (Catha edulis) chewing and orodental health: a systematic review and metaanalysis. S Afr Med J 2014;104:773-779.

3. Al-Kholani AI. Influence of khat chewing on periodontal tissues and oral hygiene status among Yemenis. Dent Res J (Isfahan) 2010;7:1-6.

4. Yarom N, Epstein J, Levi H, Porat D, Kaufman E, Gorsky M. Oral manifestations of habitual khat chewing: a case-control study. Oral Surg Oral Med Oral Pathol Oral Radiol Endod 2010;109:e60-e66.

5. Amran AG, Ataa MA. Statistical analysis of the prevalence, severity and some possible etiologic factors of gingival recessions among the adult population of Thamar city, Yemen. RSBO 2011;8:305-313.

6. Al-Sharabi AK, Shuga-Aldin H, Ghandour I, Al-Hebshi NN. Qat chewing as an independent risk factor for periodontitis: a cross-sectional study. Int J Dent 2013;2013:317640.

7. Ali AA, Al-Sharabi AK, Aguirre JM, Nahas R. A study of 342 oral keratotic white lesions induced by qat chewing among 2500 Yemeni. J Oral Pathol Med 2004;33:368-372.

8. Gorsky M, Epstein JB, Levi H, Yarom N. Oral white lesions associated with chewing khat. Tob Induc Dis 2004;2:145-150.

9. Rawal SY, Rawal YB, Anderson KM, Bland PS, Stein SH. Plasma cell gingivitis associated with khat chewing. PERIO 2008;5:21-28.

10. Ashri N, Gazi M. More unusual pigmentations of the gingiva. Oral Surg Oral Med Oral Pathol 1990;70:445-449.

11. Al-Bayaty FH, Ali NW, Bulgiba AM, Masood M, Hussain SF, Abdulla MA. Tooth mortality in khat and non khat chewer in Sana'a Yemen. Sci Res Essay 2011;6:1039-1045.

12. Luqman W, Danowski TS. The use of khat (Catha edulis) in Yemen. Social and medical observations. Ann Intern Med 1976;85:246-249.

13. Cobián OG, Triguero RJP, Pérez HS, Salgado KMR. Temporomandibular disorders in qat addicted people. Rev Cubana Estomatol 2012; 49:268-275 (Spanish).

14. Nyanchoka IN, Dimba EA, Chindia ML, Wanzala P, Macigo FG. The oral and dental effects of khat chewing in the Eastleigh area of Nairobi. J Kenya Dent Assoc 2008;1:37-42.

15. Sawair FA, Al-Mutwakel A, Al-Eryani K, Al-Surhy A, Maruyama S, Cheng J, et al. High relative frequency of oral squamous cell carcinoma in Yemen: qat and tobacco chewing as its aetiological background. Int J Environ Health Res 2007;17:185-195.

16. Al-hebshi NN, Al-ak'hali MS. Experimental gingivitis in male khat (Catha edulis) chewers. J Int Acad Periodontol 2010;12:56-62.

17. Jorgensen E, Kaimenyi JT. The status of periodontal health and oral hygiene of miraa (catha edulis) chewers. East Afr Med J 1990;67:585-
590.

18. Macigo FG, Mwaniki DL, Guthua SW. The association between oral leukoplakia and use of tobacco, alcohol and khat based on relative risks assessment in Kenya. Eur J Oral Sci 1995;103:268-273.

19. Gift HC. Oral health outcomes research: challenges and opportunities. In: Slade GD, editor. Measuring oral health and quality of life. Chapel Hill: University of North Carolina, Dental Ecology; 1997, p. 25-46.

20. Astatkie A, Demissie M, Berhane Y, Worku A. Prevalence of and factors associated with regular khat chewing among university students in Ethiopia. Subst Abuse Rehabil 2015;6:41-50.

21. Faul F, Erdfelder E, Buchner A, Lang AG. Statistical power analyses using $G^{*}$ Power 3.1: tests for correlation and regression analyses. Behav Res Methods 2009;41:1149-1160.

22. Gebreslassie M, Feleke A, Melese T. Psychoactive substances use and associated factors among Axum University students, Axum Town, North Ethiopia. BMC Public Health 2013;13:693.

23. Locker D, Miller Y. Evaluation of subjective oral health status indicators. J Public Health Dent 1994;54:167-176.

24. Kojima A, Ekuni D, Mizutani S, Furuta M, Irie K, Azuma T, et al. Relationships between self-rated oral health, subjective symptoms, oral health behavior and clinical conditions in Japanese university students: a cross-sectional survey at Okayama University. BMC Oral Health 2013;13:62.

25. White IR, Royston P, Wood AM. Multiple imputation using chained equations: issues and guidance for practice. Stat Med 2011;30:377399.

26. StataCorp. Stata multiple-imputation reference manual: release 12. College Station: Stata Press; 2011, p. 47-64, 113-159.

27. Heeringa SG, West BT, Berglund PA. Applied survey data analysis. Boca Raton: CRC Press; 2010, p. 14-51, 210-211.

28. Cameron AC, Trivedi PK. Regression analysis of count data. 2nd ed. Cambridge: Cambridge University Press; 2013, p. 69-110.

29. Klein D. Mimrgns: Stata module to run margins after mi estimate; 2014 [cited 2014 Sep 12] Available from: https://ideas.repec.org/c/ boc/bocode/s457795.html.

30. Bruch JM, Treister NS. Clinical oral medicine and pathology. New York: Humana Press; 2010, p. 43.

31. Lukandu OM, Neppelberg E, Vintermyr OK, Johannessen AC, Costea DE. Khat alters the phenotype of in vitro-reconstructed human oral mucosa. J Dent Res 2010;89:270-275.

32. Halbach H. Medical aspects of the chewing of khat leaves. Bull World Health Organ 1972;47:21-29.

33. Ali AA, Al-Sharabi AK, Aguirre JM. Histopathological changes in oral mucosa due to takhzeen al-qat: a study of 70 biopsies. J Oral Pathol Med 2006;35:81-85.

34. Date J, Tanida N, Hobara T. Qat chewing and pesticides: a study of adverse health effects in people of the mountainous areas of Yemen. Int J Environ Health Res 2004;14:405-414.

35. Rosenzweig KA, Smith P. Periodontal health in various ethnic groups in Israel. J Periodontal Res 1966;1:250-259.

36. Petersen PE. Challenges to improvement of oral health in the 21st century: the approach of the WHO Global Oral Health Programme. Int Dent J 2004;56:329-343.

37. FDI World Dental Federation. High risk of oral diseases and low access to care condemns developing countries to sub-standard oral health [cited 2014 Dec 16]. Available from: http://www.fdiworldental.org/ media/press-releases/latest-press-releases/25022014-developing-countries-condemned-to-sub-standard-oral-health.aspx. 